\title{
¿CÓMO ACTUALIZAR EL ACONTECIMIENTO? REFLEXIONES SOBRE UNA RETROSPECTIVA DE ESCOMBROS
}

\author{
HOW TO REINTERPRET THE HAPPENING? \\ REFLECTIONS ON A RETROSPECTIVE OF ESCOMBROS
}

Luciana Baez Escobar | lubaez.95@gmail.com

María Eugenia Bifaretti | meugeniabifa@gmail.com

Julián Facundo Duarte | jf.duarte@live.com

Facultad de Bellas Artes. Universidad Nacional de La Plata. Argentina

Recibido: 21/3/2018 | Aceptado: 7/7/2018

\section{RESUMEN}

En el marco de la investigación que realizamos sobre las primeras acciones del Grupo Escombros a través del archivo personal de Héctor Puppo -integrante y cofundador-, nos embarcamos en una reflexión en torno a la muestra Grupo Escombros. Poncortos 30 Años (1988-2018) realizada en la Galería Walden (CABA) de marzo a mayo de 2018. En esta reseña, indagaremos en cómo el archivo de obras efímeras y sus paratextos, constituyen una herramienta de un gran potencial para la presentación y actualización de este tipo de producciones.

\section{PALABRAS CLAVE}

Escombros; archivo; arte efímero; Galería Walden

\section{ABSTRACT}

As part of our investigation into the early works of Escombros Group through the personal archive of Hector Puppo - founder and participant- we set out to give some impressions on the retrospective exhibition Grupo Escombros. Poncortos 30 Años (1988-2018), which took place at Walden Gallery (Buenos Aires) from March to May in 2018. In this review, we will examine how the archive of the work itself and its paratext represent an important tool with great potential for the presentation and the reinterpretation of this type of productions.

\section{KEYWORDS}

Escombros; archive; ephemeral art; Walden gallery 
Pensar en una muestra retrospectiva de un grupo cuyas obras son de carácter efímero, como performances, acciones o happenings, implica enfrentarse con ciertas dificultades: ¿cómo lograr que una obra efímera trascienda temporalidades?, ¿cómo historizar la acción?, ¿cómo y con qué fin actualizar el acontecimiento? Esto es lo que nos preguntamos cuando supimos que se llevaría a cabo la exposición Grupo Escombros. Pancartos 30 años (1988-2018) en la Galería Walden, durante marzo de 2018.

Al considerar estas preguntas de manera aislada, las primeras respuestas se suelen vincular con dispositivos tecnológicos que, al alcance de la mano, permiten la posibilidad de registrar cierto suceso a toda hora y en todo lugar. Sin embargo, si nos situamos treinta años atrás, en el momento en que el Grupo Escombros realiza sus primeras acciones artísticas, rápidamente entendemos que el panorama es otro. En un principio, teniendo en cuenta el tipo de tecnologías disponibles para ello, se desprende que el material resultante tenderá a ser algo más rígido y acartonado; sin embargo, por ese mismo motivo, hay más posibilidades de pensar que su realización estuvo atravesada por una voluntad reflexiva, crítica, y más apuntada a la perdurabilidad que a la difusión inmediata, como suele pasar en la actualidad.

Poncortos fue la primera acción del grupo [Figura 1], en el año 1989, y consistió en mostrar quince fotografías de una performance que el grupo había realizado previamente en un espacio lleno de escombros.

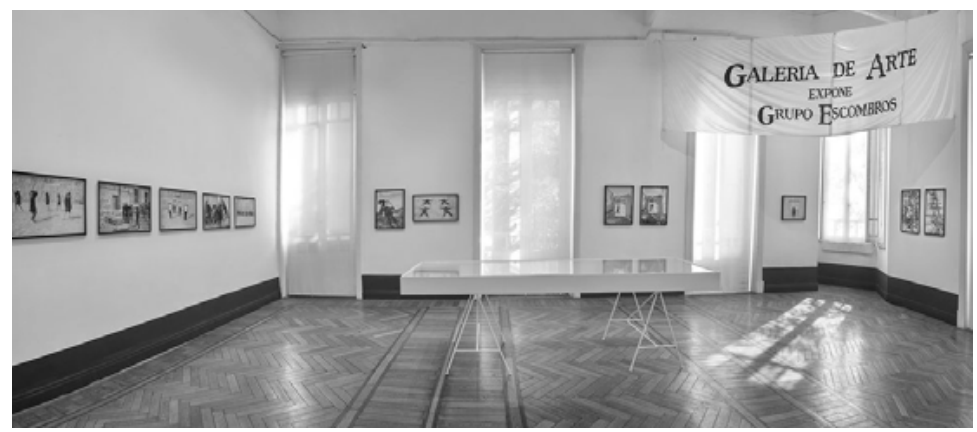

Figura 1. Pancartos (1989). Fotografía de la performance. Archivo personal de Héctor Puppo

Las presentaron a modo de pancartas clavadas en un terreno semibaldío bajo la autopista Buenos Aires-La Plata (en Paseo Colón y Cochabamba, barrio de San Telmo) y luego de dos horas de exhibición las hicieron circular por las calles en una manifestación colectiva que cortaba el tránsito. La acción incluyó un cartel que decía «Galería de Arte: Expone Grupo Escombros», una mesa con gaseosas y vino para el público, una tarjeta de invitación y el catálogo con su primer manifiesto, Lo estético de lo roto (1989). 
Al entender la condición de lo efímero que el grupo manejaba sobre lo artístico en sus acciones más tempranas como Poncortos, podemos pensar en dos posibles maneras de actualizarla. Una de ellas podría consistir en hacer una representación 0 reconstrucción lo más parecida posible a lo sucedido, con un gesto artificioso y forzado; otra, en dar cuenta del sistema narrativo en el que fue producida, a través de la presentación de los paratextos que la rodean. Cuando entramos a la exposición Grupo Escombros. Pancortos 30 años (1988-2018) comprendimos que la operación efectuada fue la segunda. No optaron por una representación de la obra-acontecimiento -que podría tener sentido para recrear experiencias de obras de carácter más lúdico o hedonista-, sino que se exhibió una presentación de su universo discursivo, aquel que se inscribe en el archivo del grupo. De esta manera, la exposición presenta los registros y fragmentos del acontecimiento: un audiovisual, las fotografías enmarcadas en las paredes, el cartel, recortes de diario, notas de prensa, la tarjeta de invitación a la obra, el manifiesto y bocetos que dan cuentan del proceso [Figura 2].

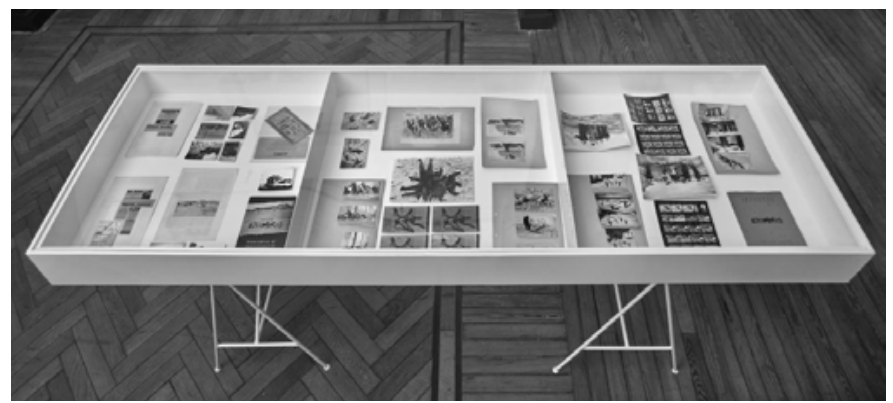

Figura 2. Grupo Escombros. Pancortas 30 años (1988-2018) (2018). Vista de la exposición. Galería Walden

Fiel al carácter efímero de la obra, la exposición no intenta crear una atmósfera ilusoria en la que el público adquiere la experiencia del acontecer de la intervención, sino que -quizás de una forma menos llamativa, pero no por ello menos interesante- expone los elementos paratextuales que hoy permiten afirmar que la obra sucedió. Siguiendo a Gérard Genette (2001), estos elementos cumplen ese mismo objetivo: rodean al texto, la obra, y lo prolongan «precisamente por presentarlo, en el sentido habitual de la palabra, pero también en su sentido más fuerte: por darle presencia, por asegurar su existencia en el mundo» (p. 7). De esta manera, podemos comprender que lo que permite que Poncartos sea sostenida en el tiempo y actualizada históricamente son tanto sus paratextos como el archivo que los estructura: aquel sistema de funcionamiento que determina el discurso sobre los acontecimientos, que marca las reglas de lo que puede ser dicho sobre ellos (Foucault, 1979). 
Es ineludible destacar que la actualización de Poncartos mediante este sistema discursivo no sucede en un momento azaroso, sino que se realiza, intencionalmente, en una fecha particular: el trigésimo aniversario del Grupo Escombros y se inauguró un 24 de marzo, Día Nacional de la Memoria por la Verdad y la justicia. Esta fecha especial guarda relación directa con las motivaciones iniciales del grupo y con el compromiso que tuvo desde sus inicios con la perdurabilidad de la memoria reciente y pone particular énfasis en la situación del país tras los años de la dictadura, las atrocidades cometidas por los militares durante este periodo, y el clima social generado. En el catálogo que se presentó con la muestra en el año 1989, se hacen presentes esos lineamientos que el grupo sostenía, donde se explicita el carácter casi historiográfico de sus obras. A su vez, estas ideas son retomadas en el material entregado en la exposición de la Galería Walden, citado por María de los Ángeles De Rueda (2018) en su texto acerca de Poncortos: «Expresamos lo roto, lo quebrado, lo violado, lo vulnerado, lo despedazado. Es decir, el hombre y el mundo de aquí y ahora» (s/p), acompañado por un breve recorrido histórico del grupo.

El hecho de generar estos cruces temporales resulta un gesto poético y, al mismo tiempo, pone en evidencia el interesante potencial del archivo y sus paratextos en las prácticas curatoriales contemporáneas. El acontecimiento, la acción, la obra efímera resultan, así, historizados, pero no de una forma hierática, sino activa; se muestran comprometidos con el presente con el que se los hace dialogar y amplían las posibilidades de lo que puede ser dicho acerca de ellos.

Otro aspecto que encontramos interesante en este diálogo de temporalidades es que los parámetros estéticos que mantiene la exposición remiten a aquella estética que el grupo explica en su manifiesto Lo estética de lo roto (1989): el no-color, el uso excluyente del blanco y negro y una austeridad en lo plástico acentúan la potencia de lo visual, muestran la gravedad y lo urgente de los mensajes por delante de lo espectacular [Figura 3].

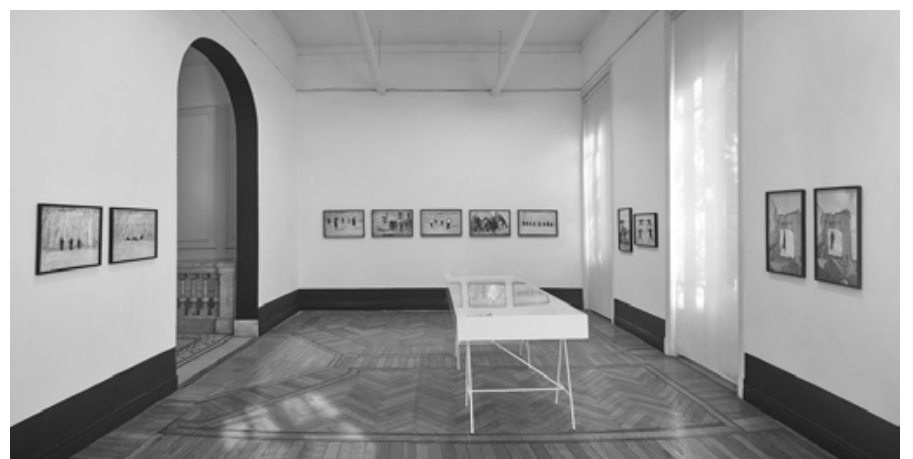

Figura 3. Grupo Escombros. Pancartas 30 años (1988-2018) (2018). Vista de la exposición. Galería Walden 
Asimismo, el hecho de que se omita la figura de curador evidencia la fidelidad a la idea de Escombros de ser un grupo abierto y horizontal, donde el yo se reemplaza por el nosotros (Grupo Escombros, 1989), tanto al interior del grupo y las obras como en relación con la totalidad de la sociedad.

Sin embargo, siguiendo el manifiesto, encontramos que hay cierto repudio a la historización: «El sueño del Poder es congelar la historia. El artista alerta a la conciencia colectiva cada vez que cae prisionera de ese sueño», y un rechazo al pasado: «El pasado está muerto y su destino es convertirse en polvo. El presente está vivo y su destino, como el de la vida, es crecer, reproducirse e imponerse» (Grupo Escombros, 1989, s/p).

Cabe preguntarse, entonces, si la exposición pone en tensión este fuerte ideal del grupo por mantenerse en un puro presente, y repudia cualquier tipo de vuelta en el tiempo. Nos aventuramos a afirmar que no, ya que consideramos que el pasado que Escombros propone dejar de lado hace referencia a la historia hegemónica, esa escrita por los vencedores, que intentan cristalizarla para perpetuar su dominio sobre las clases subalternas. Sus acciones actualizadas en el presente sugieren, más bien, repreguntar por aquellos conflictos que en el discurso de la historia oficial aparecen, aparentemente, superados y, así, deconstruir nuestro propio pasado. En este sentido, estas inquietudes se escapan del dominio de los y las especialistas e interpelan a la sociedad en su totalidad.

Así, teniendo en cuenta la posibilidad de actualización mencionada anteriormente, consideramos que las proposiciones del grupo trascienden la dimensión temporal de sus momentos de creación. Podemos afirmar que este fenómeno se da porque los mecanismos y las estructuras de poder contra las que Escombros se proclamó en sus inicios siguen arraigadas en nuestras construcciones sociales en la actualidad, lo que nos lleva a vernos interpelados e interpeladas por sus obras al día de hoy, con la misma intensidad con la que fueron experimentadas hace tres décadas.

\section{REFERENCIAS}

De Rueda, M. A. (2018). Grupo Escombros/Poncortos. Recuperado de http:// www.waldengallery.com/exhibiciones/\#/new-gallery/

Foucault, M. (1979). La Arqueología del Saber. Ciudad de México, México: Siglo XXI. 
Genette, G. (2001). Umbrales. Ciudad de México, México: Siglo XXI.

Grupo Escombros (1988). Pancortos [Acción]. Recuperado de http://www. grupoescombros.com.ar/pancartas.html

Grupo Escombros (1989). Lo estética de lo roto. Primer Monifiesto. Recuperado de http://www.grupoescombros.com.ar/imgs/publicaciones/manifiestos/laesteticadeloroto.html 